\title{
Microenvironment Changes (in pH) Affect VEGF Alternative Splicing
}

\author{
Ana Paula Elias • Sergio Dias
}

Received: 10 April 2008 / Accepted: 4 July 2008 / Published online: 29 July 2008

(C) Springer Science + Business Media B.V. 2008

\begin{abstract}
Vascular endothelial growth factor-A (VEGF-A) has several isoforms, which differ in their capacity to bind extracellular matrix proteins and also in their affinity for VEGF receptors. Although the relative contribution of the VEGF isoforms has been studied in tumor angiogenesis, little is known about the mechanisms that regulate the alternative splicing process. Here, we tested microenvironment cues that might regulate VEGF alternative splicing. To test this, we used endometrial cancer cells that produce all VEGF isoforms as a model, and exposed them to varying $\mathrm{pH}$ levels, hormones, glucose and $\mathrm{CoCl}_{2}$ (to mimic hypoxia). Low $\mathrm{pH}$ had the most consistent effects in inducing variations in VEGF splicing pattern (VEGF121 increased significantly, $p<0.001$, when compared to VEGF145, 165 or 189). This was accompanied by activation of the p38 stress pathway and SR proteins (splicing factors) expression and phosphorylation. SF2/ASF, SRp20 and SRp40 down-regulation by siRNA impaired the effects
\end{abstract}

Electronic supplementary material The online version of this article (doi:10.1007/s12307-008-0013-4) contains supplementary material, which is available to authorized users.

\footnotetext{
A. P. Elias $\cdot$ S. Dias $(\bowtie)$

Angiogenesis Laboratory,

Centro de Investigação em Patobiologia Molecular (CIPM),

Instituto Português de Oncologia de Francisco Gentil,

Lisboa, Portugal

e-mail: sergidias@ipolisboa.min-saude.pt

S. Dias

Instituto de Medicina Molecular,

Lisboa, Portugal

S. Dias

Instituto Gulbenkian de Ciência,

Oeiras, Portugal
}

of $\mathrm{pH}$ stimulation, blocking the shift in VEGF isoforms production. Taken together, we show for the first time that acidosis (low $\mathrm{pH}$ ) regulates VEGF-A alternative splicing, may be through p38 activation and suggest the possible SR proteins involved in this process.

Keywords Alternative splicing - Endometrial carcinoma . Microenvironment $\cdot$ SR proteins $\cdot$ VEGF

$\begin{array}{ll}\text { Abbreviations } \\ \text { ARE } & \text { AU-rich elements } \\ \text { DMEM } & \text { Dulbecco's modified Eagle's medium } \\ \text { ECL } & \text { enhanced chemiluminescence } \\ \text { ECM } & \text { extracellular matrix } \\ \text { ELISA } & \text { enzyme-linked immunosorbent assay } \\ \text { ERK } & \text { extracellular signal-regulated kinase } \\ \text { FBS } & \text { fetal bovine serum } \\ \text { hnRNP } & \text { heterogeneous nuclear ribonucleoprotein } \\ \text { HuR } & \text { hypoxia-induced stability factor } \\ \text { MAPK } & \text { mitogen-activated protein kinase } \\ \text { PAIP2 } & \text { poly(A)-binding protein-interacting protein } 2 \\ \text { RQ-PCR } & \text { real time RT-PCR } \\ \text { RRM } & \text { RNA recognition motif } \\ \text { RS domain } & \text { domain rich in alternating serine and } \\ & \text { arginine residues } \\ \text { SAPK/JNK } & \text { stress-activated protein kinase/ } \\ \text { Sun-amino-terminal kinase } \\ \text { SDS-PAGE } & \text { sodium dodecyl sulphate-polyacrylamide gel } \\ & \text { electrophoresis } \\ \text { siRNA } & \text { small interfering RNA } \\ \text { SRp } & \text { serine/arginine-rich protein } \\ \text { UTR } & \text { untranslated region } \\ \text { VEGF } & \text { vascular endothelial growth factor }\end{array}$




\section{Introduction}

Angiogenesis is important for the expansion of solid and hematologic cancers. In response to metabolic changes occurring within the tumor microenvironment, such as increased hypoxia and acidosis [1], a cascade of events takes place, resulting in the production of angiogenic "promoters" (stimulators) and a decrease in angiogenesis inhibitors [2-4]. One of the best known angiogenic stimulators, produced by most tumors, is the vascular endothelial growth factor (VEGF-A).

The VEGF-A gene undergoes alternative splicing between exons 5 and 8 (Fig. 1) [5]. This mechanism results in the predominant production of four isoforms, which differ in molecular weight, and are thus known as VEGF121, 165, 189, and 145. VEGF165 is the predominant secreted isoform, produced by most cell types (and most tumors), and although it is a diffusible protein, a significant fraction binds to the extracellular matrix (ECM). In contrast, VEGF121 is a freely diffusible isoform that does not bind to heparin, while VEGF189 binds strongly to heparin and therefore is completely sequestered in the ECM. The isoform 145 of VEGF is observed preferentially in carcinomas of the female reproductive system [6]. The importance of selective VEGF isoform secretion by tumors has been demonstrated [7]. In detail, tumors that secrete predominantly the VEGF121 isoform have increased dilated and peripheral blood vessels, while tumors overexpressing VEGF189, had highly branched and internal neo-vasculature [8]. More recently, increased ratio of VEGF121 versus the 165 or 189 isoforms was shown to be critical for the angiogenic phenotype of prostate cancers [9].
VEGF-A is produced by cells under stress, such as during hypoxia, resulting in tissue angiogenesis and oxygenation, although the molecular mechanisms regulating VEGF production in response to microenvironmental stimuli other than hypoxia, such as acidosis, are still poorly characterized [10].

Alternative splicing is a major mechanism for modulating the expression of cellular and viral genes and enables a single gene to increase its coding capacity. The VEGF isoforms mentioned above represent one family of proteins whose expression may be regulated by alternative splicing.

The family of SR (serine/arginine-rich) proteins has been implicated in splicing; they are characterized by an RNA recognition motif (RRM) and a C-terminal domain rich in alternating serine and arginine residues (the RS domain) [11]. The RRMs determine RNA binding specificity, whereas the RS domain mediates protein-protein interactions that are thought to be essential for the recruitment of the splicing apparatus and for the splice site pairing.

In the present report, we studied the influence of microenvironment cues that could affect the VEGF-A gene splicing pattern, and determined the molecular mechanisms involved.

\section{Results}

Microenvironment Changes Affect VEGF Alternative Splicing Pattern

We investigated how changes in the microenvironment might affect the pattern of VEGF alternative splicing (Fig. 1), using endometrial carcinoma cells as a model

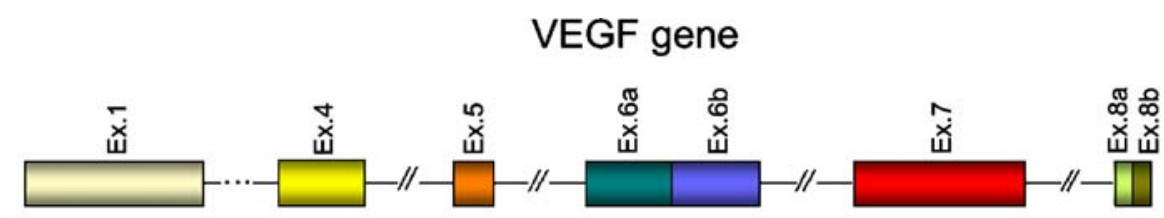

\section{VEGF transcripts}

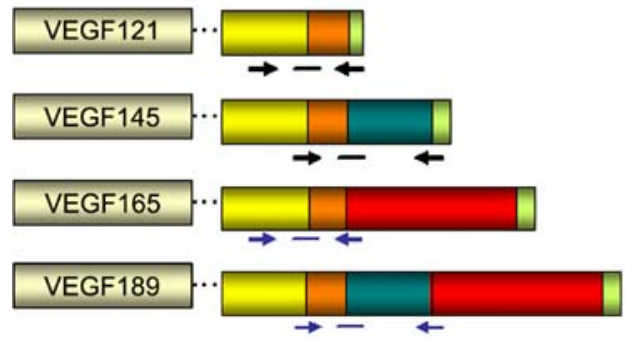

Fig. 1 VEGF isoforms that result from pre-mRNA alternative splicing. These isoforms differ in the presence or absence of exons 6 and 7 that codes for heparin-binding domains and in the presence of exon $8 \mathrm{a}$ or $8 \mathrm{~b}$ that induce antagonist effects of VEGF. The localization

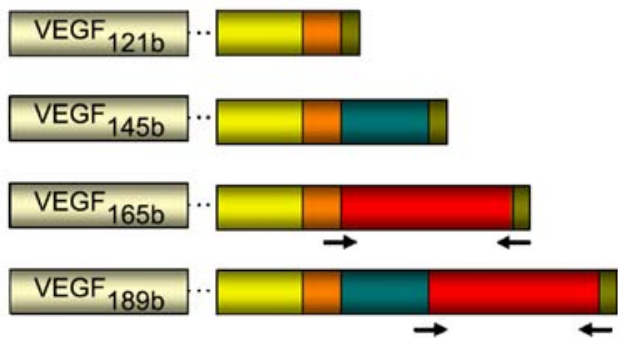

of the probes and primers used to amplify the different isoforms of VEGF are indicated by a line or an arrow, respectively. The lines and arrows in blue represent the probes and primers used to amplify either VEGF165+VEGF165b or VEGF189+VEGF 189 b 
(since these cells express all VEGF-A isoforms). For this purpose, we induced changes in the culture medium (by exposing the cells to acidic $\mathrm{pH}$, progesterone, $\beta$-estradiol, glucose and cobalt chloride, to mimic for hypoxia), and quantified the ratio of VEGF isoforms by real time RT-PCR (RQ-PCR). As expected, hypoxia significantly increased VEGF production, as did acidosis (Fig. 2a,b and Supplementary Fig. 1). However, a more evident shift in the pattern of VEGF isoforms produced, occurred in samples subjected to lower $\mathrm{pH}$. A pH 5.5 induced a preferential VEGF121 increase $(p<0.001)$, suggesting it modulated the mechanisms involved in alternative splicing.

The VEGF $_{x x x} b$ isoforms, previously shown to be involved in the regulation of tumor angiogenesis [12], were expressed at very low levels by the cell line used in our studies. Notably, in this study $\mathrm{VEGF}_{\mathrm{xxx}} \mathrm{b}$ isoforms did not accompany the variations observed in VEGFxxx isoforms $\left(\mathrm{VEGF}_{165} \mathrm{~b}\right.$ and $\mathrm{VEGF}_{189} \mathrm{~b}$ did not differ significantly from control to $\mathrm{pH} 5.5$ conditions, $p>0.05$ ). Therefore we concluded that the modifications in VEGF alternative

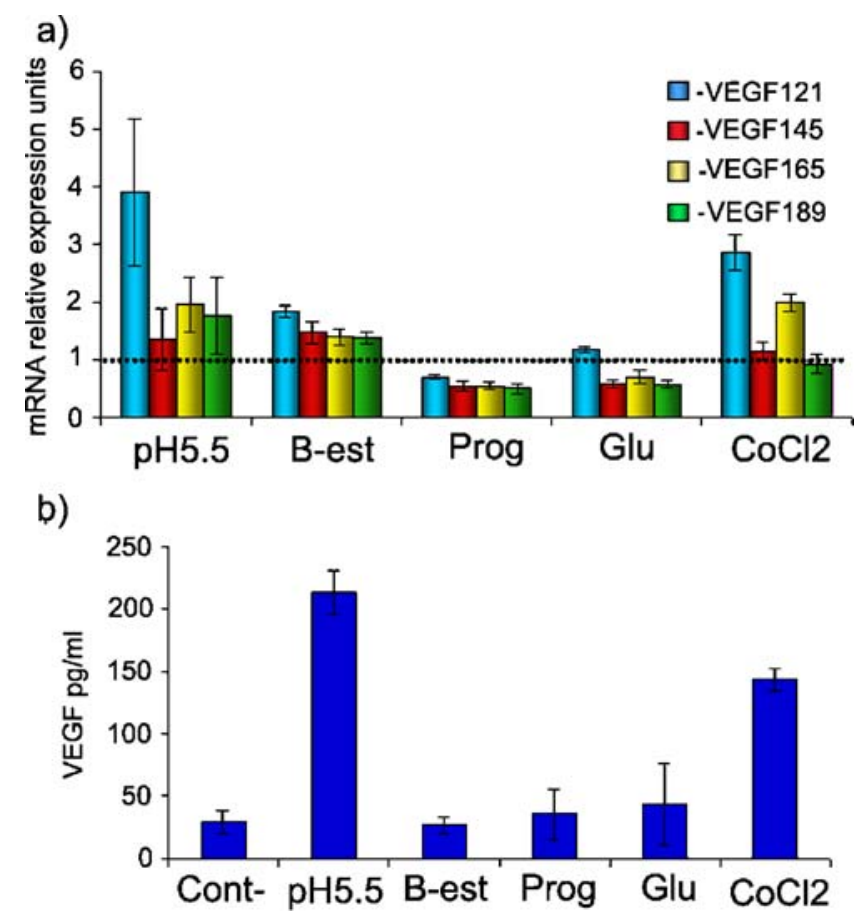

Fig. 2 VEGF isoforms expression pattern by RL95 cells in response to changes in the microenvironment. By real time RT-PCR (a) and ELISA (b), we can see an increase in VEGF production in acidic and hypoxic (mimicked by $\mathrm{CoCl}_{2}$ ) conditions. A shift in the VEGF isoforms splicing pattern is more evident at $\mathrm{pH} 5.5$ where VEGF121 expression is significantly different from all other isoforms $(p<0.001)$. In hypoxic conditions VEGF121 is also significantly different from VEGF145 and $189(p<0.001)$ and from VEGF165 $(p<0.05)$. In a the level of each isoform in different conditions is represented relatively to control conditions (which is considered equal to 1 ). In graph a $n=10$ and in graph $\mathbf{b} n=3$ splicing observed in low $\mathrm{pH}$, did not involve the $\mathrm{VEGF}_{\mathrm{xxx}} \mathrm{b}$ alternative transcripts (Fig. 3).

The shift in VEGF isoform ratio occurred within $6 \mathrm{~h}$ of $\mathrm{pH} 5.5$, but the greatest difference was seen after $8 \mathrm{~h}$ of stimulation (Fig. 4a).

VEGF Isoform Shift in Acidic $\mathrm{pH}$ is Accompanied by $\mathrm{p} 38 / \mathrm{MAPK}$ Activation

Since acidic $\mathrm{pH}$ induced a shift in isoform production by RL95 cells, next we sought to define the signaling pathways that might be involved in this effect.

As shown in Fig. 4b, $8 \mathrm{~h}$ in acidic $\mathrm{pH}$ induced the activation of the stress signaling pathways p38 MAPK $(p<0.05)$ and $\mathrm{SAPK} / \mathrm{JNK}$, while ERK and Akt remained unchanged. In vitro blockade of the two signaling pathways using specific inhibitors demonstrated that cells cultured in the presence of the p38 pathway inhibitor (SB202190) did not respond to the acidic $\mathrm{pH}$ (Fig. 4c). Under these conditions the shift in VEGF isoform production was not observed (VEGF121 was not significantly different from all the other isoforms, $p>0.05$ ), while the SAPK/JNK inhibitor SP600125 had little effect (VEGF121 vs VEGF165, $p<$ 0.001 , as observed in $\mathrm{pH} 5.5$ condition). These data indicate that the p38 stress signaling pathway may be involved in the effects of acidic $\mathrm{pH}$ that result in modulation of the VEGF alternative splicing pattern.

SR Proteins Could be Involved in the Regulation of VEGF Isoforms

SR proteins have been described to be involved in the control of constitutive and alternative splicing of genes [11]. To evaluate which SR proteins could be activated by the p38 stress signaling pathway and involved in VEGF isoform shift in acidic conditions, the modulation of SR proteins was investigated.

Using an antibody against phosphorylated-SR proteins, we verified that the up-regulation of these proteins correlates with activation of the p38 signaling pathway and the shift in VEGF isoform production (Fig. 5a). Since these proteins can shuttle between the nucleus and cytoplasm we confirmed that the majority of these proteins were localized at the nucleus in $\mathrm{pH} 5.5$ (data not shown) and could be acting as splicing regulators.

By real time RT-PCR we quantified the mRNA of different SR proteins (SF2/ASF, SRp20 and SRp40) and observed that $\mathrm{pH} 5.5$ induced a significant up-regulation $(p<0.05)$ of SRp20 that could be partially inhibited in the presence of SB202190, the p38 MAPK inhibitor (Fig. 5b). This result indicates that $\mathrm{p} 38$ MAPK was not acting at the SR proteins mRNA levels and could have a role at mRNA translation or SR proteins activation (protein phosphoryla- 
Fig. 3 Discrimination between VEGFxxx and VEGF $_{x x x}$ b isoforms expression in acidic $\mathrm{pH}$. In $\mathrm{pH} 5.5$ there was a significant increase in VEGF165 $(p<0.001)$ and $189(p<0.01)$ but the expression of VEGF165b $(p<$ $0.001)$ and $189 \mathrm{~b}(p<0.001)$ is too low when compared respectively to the VEGF165 and 189 and do not change in acidic conditions $(p>0.05$ for both isoforms). The graphs represent the mean of three experiments

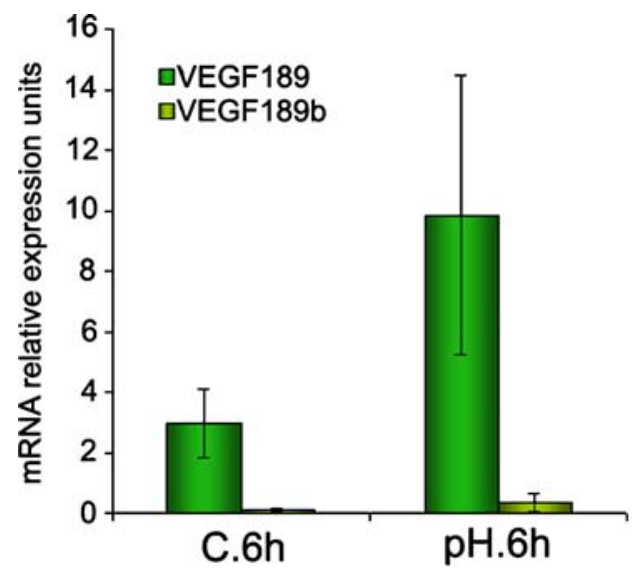

tion). To reveal the involvement of selective SR proteins in VEGF alternative splicing we used siRNA against each SR protein and tested the effect in the pattern of VEGF isoform production. siRNA against SF2/ASF, SRp20 or SRp40 reduced the production of the VEGF121 observed in $\mathrm{pH} 5.5$ (in fact, the VEGF isoforms pattern in these conditions is equal to the control), suggesting that not only we have prevented VEGF expression but also have prevented the shift in the VEGF isoforms pattern ( $p>0.05$; Fig. $6 \mathrm{~b}$ ). Thus, SF2/ASF, SRp20 and SRp40 may have a role at the VEGF alternative splicing process. These three proteins might also have a role in the alternative slicing of a VEGF regulator since by its down-regulation we affected the VEGF expression.

Since these proteins have RNA recognition motifs, we used bioinformatics software to search putative binding a)

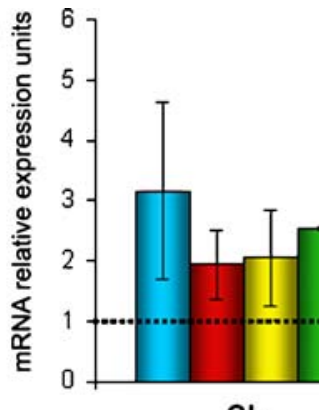

$6 \mathrm{~h}$

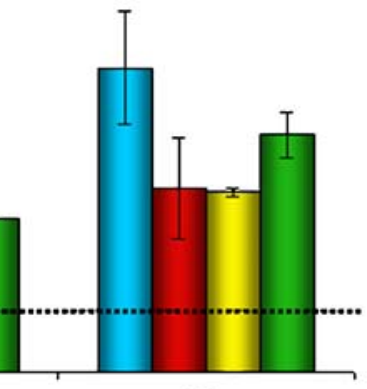

$8 \mathrm{~h}$ c)

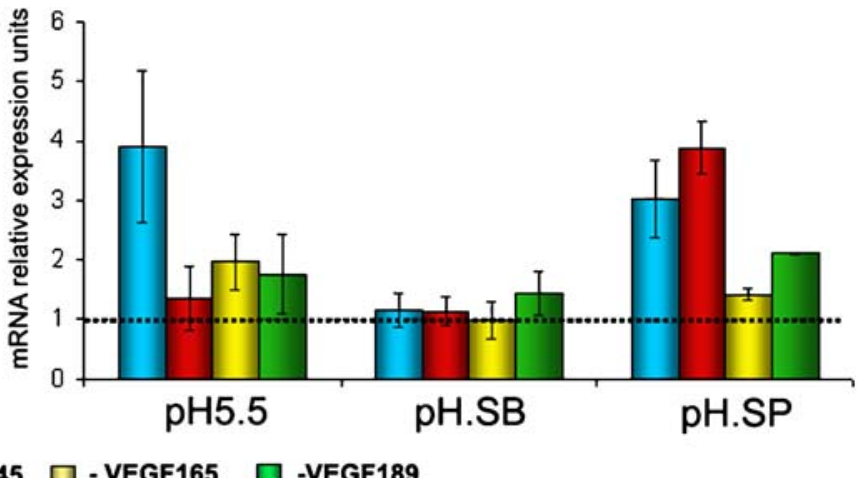
-VEGF165 $\square$-VEGF189

$\square$-VEGF121 $\square$-VEGF145

b)

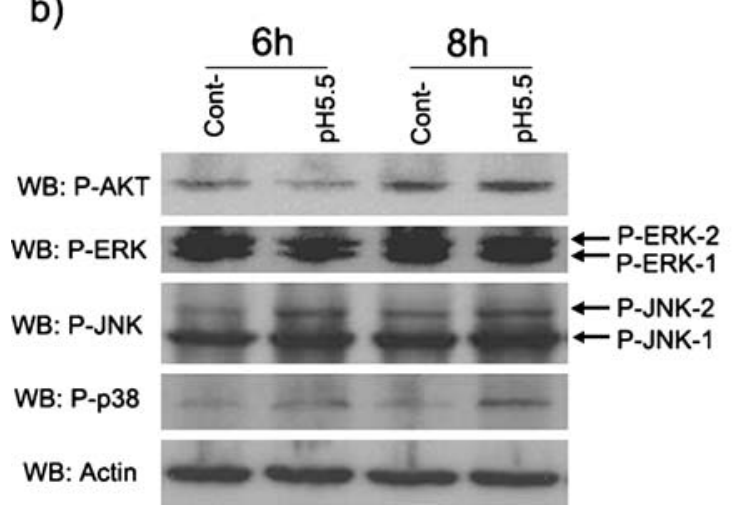

Fig. 4 a Correlation between VEGF isoforms pattern inversion and the activation of stress signaling pathways in acidic conditions. After $8 \mathrm{~h}$ of $\mathrm{pH} 5.5$ stimulation, the changes in VEGF isoform splicing pattern are more pronounced. b As shown by western blotting and densitometry quantification, at this time point, from the several signaling pathways analyzed, only the p38 MAPK $(p<0.05)$ and the SAPK/JNK stress pathways were activated. c SB202190 and
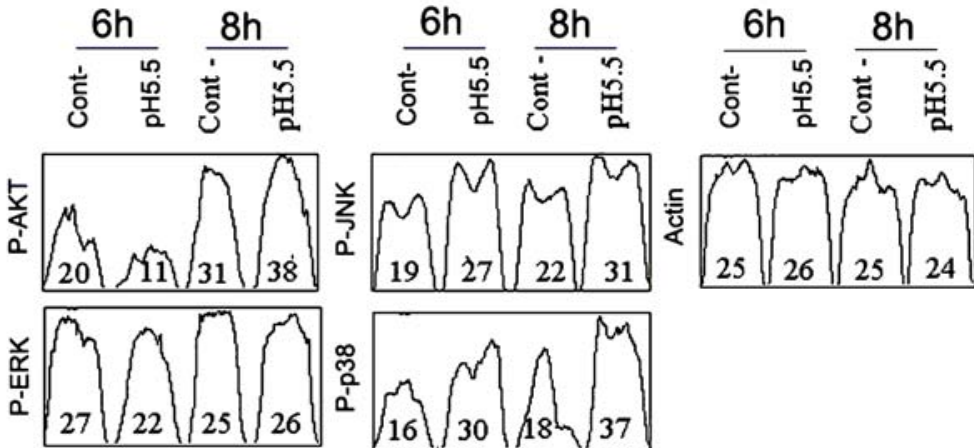

SP600125 were used to inhibit p38 MAPK and SAPK/JNK stress pathways, respectively. In the presence of the p38 inhibitor, low $\mathrm{pH}$ failed to induce a shift in VEGF splicing pattern (all VEGF isoforrms are similar, $p>0.05$ ), while the SAPK/JNK inhibitor could not prevent the shift in VEGF isoforms (VEGF121 is significantly different from VEGF165, $p<0.001)$. Graphs a and $\mathbf{c}$ represent the mean of three experiments and the blot was repeated three times 
Fig. 5 a SR proteins involved in the control of the VEGF splicing pattern. Most SR proteins were up-regulated in acidic conditions both after 6 and $8 \mathrm{~h}$ of exposure. b Expression of the SR protein SRp20, in RL95, increased significantly $(p<0.05)$ in acidic conditions and was only partially inhibited in the presence of the $\mathrm{p} 38 \mathrm{MAPK}$ inhibitor SB202190. In graph b the error bars represent the standard deviation of three independent experiments
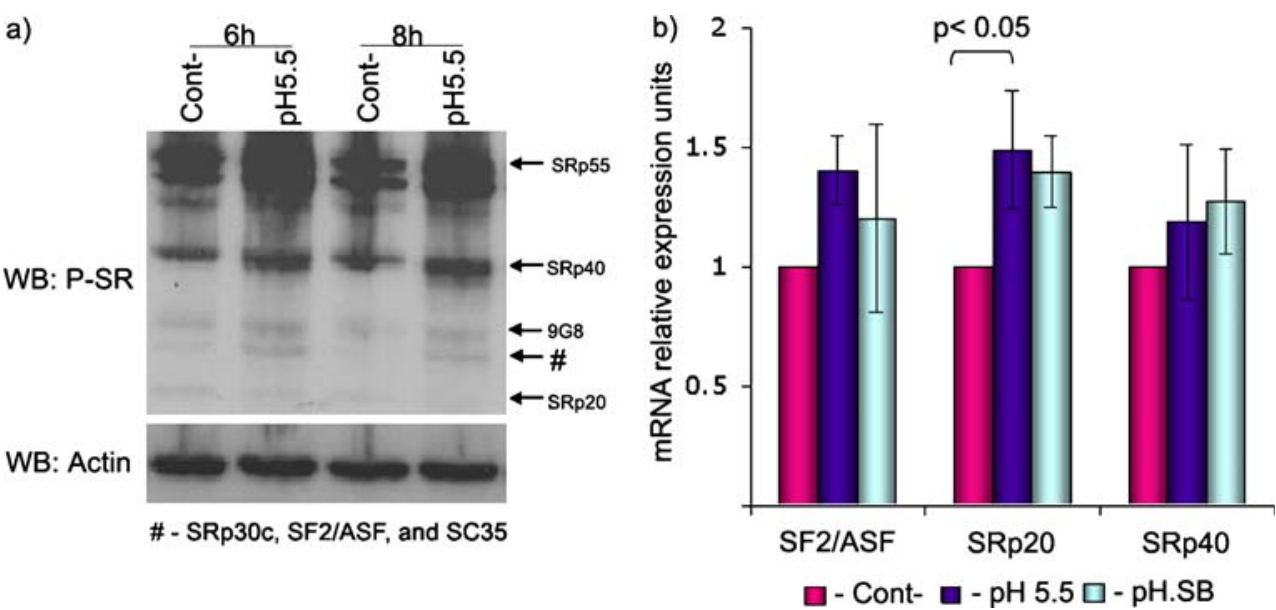

sites for these specific SR proteins, SF2/ASF, SRp20 and SRp40. This approach showed that SRp20 and SRp40 do not have putative binding sites at the exons of VEGF that are involved in alternative splicing, but SF2/ASF has putative binding sites at exons 5,7 and 8 of VEGF. Therefore, we may speculate that the SF2/ASF protein may act on alternative splicing of VEGF by binding directly to the VEGF sequence and in contrary, the SRp20 and SRp40 could be exon-binding independent by recruiting splicing factors through its serine/arginine rich domain (SR proteins property already described by Wu and Maniatis in 1993) [13].

Since SR proteins are large families of proteins involved in the global mechanisms of alternative splicing, a more detailed study must be done in order to identify other SR or hnRNP (SR proteins antagonists, heterogeneous nuclear ribonucleoproteins) proteins that may regulate the VEGF isoforms pattern in acidic conditions.
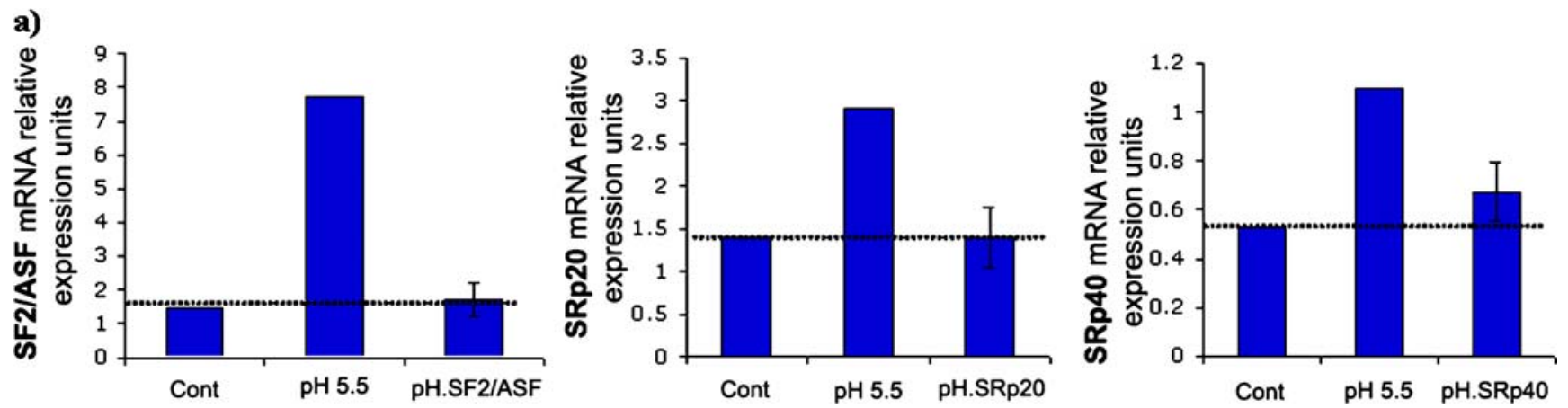

b)
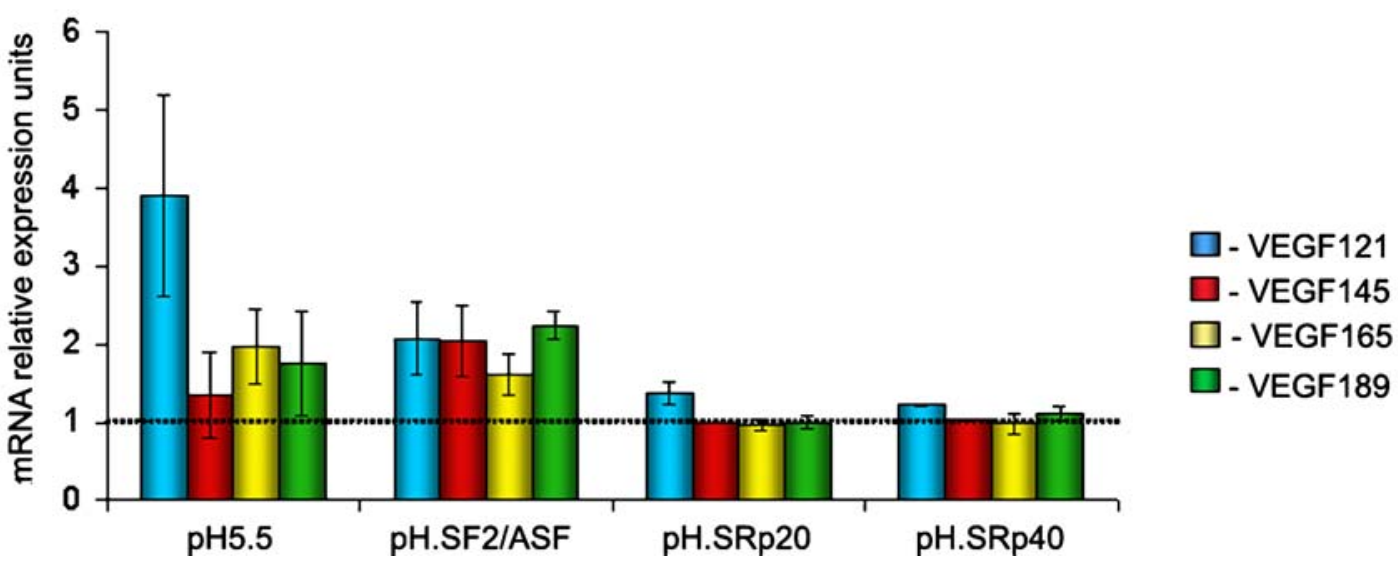

Fig. 6 a Effect of SR proteins down-regulation in VEGF alternative splicing in acidic conditions. siRNA for SF2/ASF, SRp20 and SRp40 (performed in triplicate) were used and its efficacy was demonstrated by real time RT-PCR. b Using siRNA for SF2/ASF, SRp20 and

SRp40, there was no shift in the VEGF isoforms pattern observed in acidic conditions, since all isoforms were similar to each other $(p>$ $0.05)$ and to the control $(p>0.05)$ 
Taken together, we propose a model where the activation of the p38 stress signaling pathway in response to a microenvironment signal (such as a decrease in $\mathrm{pH}$ levels) accompanies a change in VEGF alternative splicing and an increase in alternative splicing factors phosphorylation (SR proteins; see our proposed model in Fig. 7).

\section{Discussion}

Angiogenesis is an essential property of all tumors, allowing tumor expansion and contributing towards metastasis dissemination. Since it is a common feature of most malignancies, the importance of angiogenesis as a therapeutic target has been well documented [14]. Regarding the molecular signals that control the tumor angiogenesis process, the increased production of pro-angiogenic factors by most tumors has received great attention, most notably the recognition of VEGF-A as a key angiogenic factor for the majority of tumors [15]. VEGF-A has several isoforms, whose importance in the context of tumor angiogenesis has already been addressed. Although VEGF-A isoforms can bind differently to the ECM, they have also been attributed with different capacity to modulate the tumor vasculature $[8,16]$. In detail, VEGF 121 has been described as more angiogenic and tumorigenic in breast [17] and prostate [9] cancer and also to specifically improve oxygenation in experimental breast tumors [18], while VEGF189 contributes to the establishment of distant metastasis of pulmonary adenocarcinoma [19]. Given the lack of mechanistic information concerning the regulation of the VEGF alternative splicing process, in the present report we hypothesized that cues in the tumor microenvironment might selectively affect the VEGF splicing pattern, and studied the mechanisms involved in this effect.

Tumor microenvironment signals such as hypoxia and acidosis have been suggested to play a major role in the control of VEGF-A production, and consequently in modulation of angiogenesis [20]. In fact, hypoxia and acidosis are common features of the majority of solid and hematologic malignancies, perhaps as a consequence of the tumor metabolic needs, or as a consequence of an altered (pro-malignant) microenvironment. Importantly, the extracellular $\mathrm{pH}$ has been recognized as an inducer of VEGF, and also to regulate the VEGF interactions with different cells and with components of the extracellular matrix [21].

In the present study, acidosis consistently affected the VEGF alternative splicing pattern produced by endometrial cancer cells (used as a model); this correlated to a shift in the usual VEGF isoform expression pattern, resulting in an significant increase in the VEGF121, that was not accompanied by the other VEGF isoforms.

The involvement of signaling pathways and splicing machinery had not been studied in the context of VEGF alternative splicing. In our report, we reveal the involvement of p38 and possible members of the SR protein family in this mechanism. We now intend to perform a more detailed characterization of the splicing machinery involved in the VEGF alternative splicing in tumor cells exposed to different microenvironment cues.

Importantly, in addition to alternative splicing regulation, the levels of each isoform of VEGF can also be modulated

Fig. 7 Proposed model. When endometrial cancer cells are exposed to acidic medium the $\mathrm{p} 38$ MAPK signaling pathway is activated and an increase in SR proteins phosphorylation that will eventually regulate the VEGF alternative splicing takes place. In acidosis, not only VEGF expression is increased but also a modification of the alternative splicing occurs, where an increase in the proportion of VEGF121 versus VEGF145, 165, and 189 is observed
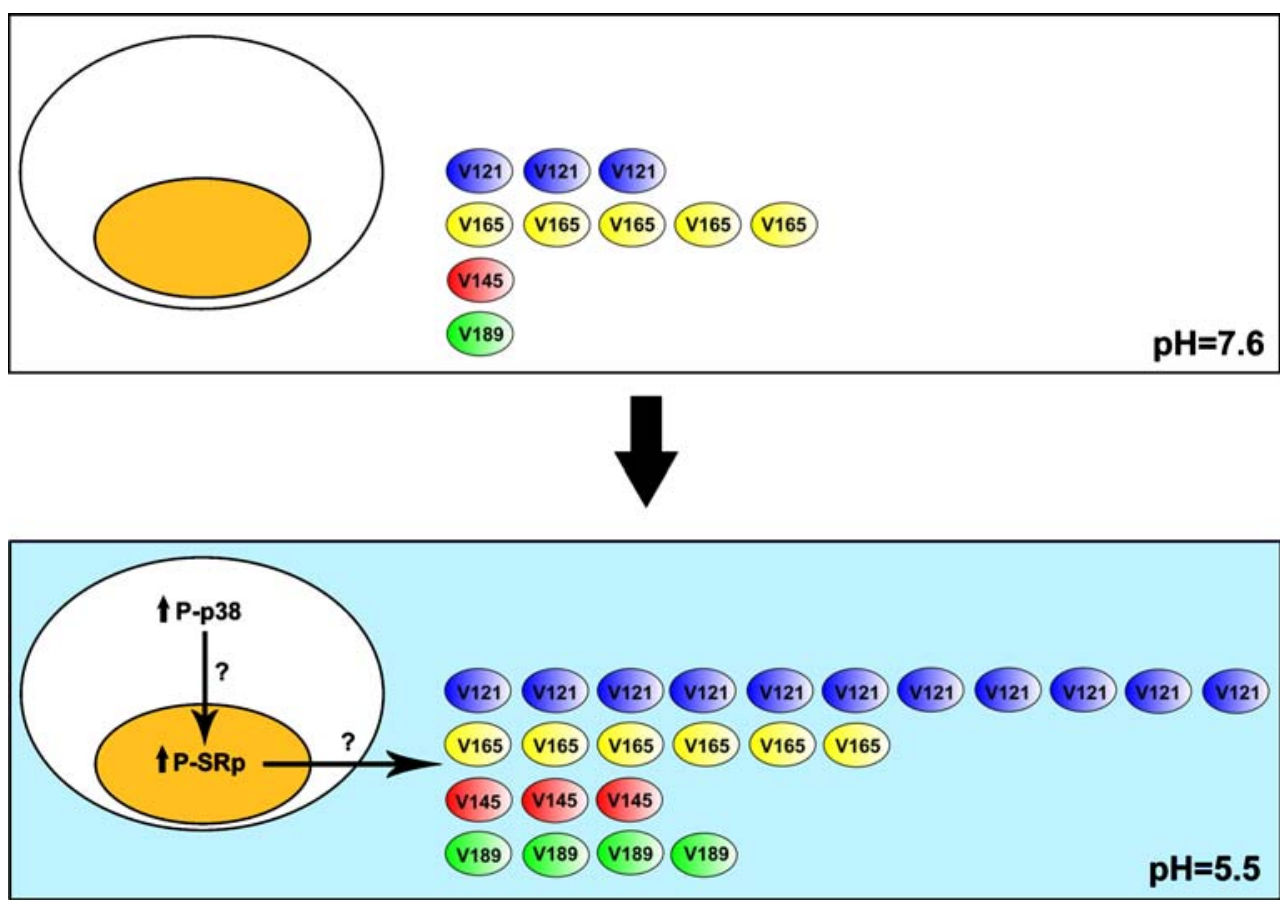
Table 1 Primers and probes used in real time RT-PCR for VEGF isoforms

\begin{tabular}{llll}
\hline VEGF isoforms & Probes $\left(6-F A M-5^{\prime} \rightarrow 3^{\prime}\right.$-TAMRA) & Primers $\left(5^{\prime} \rightarrow 3^{\prime}\right)$ & \\
\hline 121 & ACAGCACAACAAATGTGAATG & Forward & CCAGCACATAGGAGAGATGAGCTT \\
& CAGACCAAA & Reverse & CGGCTTGCACATTTTCTTGTC \\
145 & AGAGCAAGACAAGAAAAAAAA & Forward & AATGTGAATGCAGACCAAAGAAAG \\
& TCAGTTCGAGGAA & Reverse & CACATACGCTCCAGGACTTATACC \\
$165+165 \mathrm{~b}$ & ACAGCACAACAAATGTGAATG & Forward & CCAGCACATAGGAGAGATGAGCTT \\
& CAGACCAAA & Reverse & AGGCCCACAGGGATTTCTT \\
$189+189 \mathrm{~b}$ & AGAGCAAGACAAGAAAAAAAA & Forward & AATGTGAATGCAGACCAAAGAAAG \\
& TCAGTTCGAGGAA & Reverse & AGGGAACGCTCCAGGACTTATA \\
$165 \mathrm{~b}$ & - & Forward & AAGAAAATCCCTGTGGGCCTT \\
$189 \mathrm{~b}$ & - & Reverse & TGGTGAGAGATCTGCAAGTACGTT \\
& & Forward & CTGGAGCGTTCCCTGTGG \\
& & Reverse & TGGTGAGAGATCTGCAAGTACGTT \\
\hline
\end{tabular}

at the mRNA stability level. Pagès et al. [22] demonstrated that anisomycin (a strong activator of stress-activated protein kinases, SAPKs) increased VEGF mRNA stabilization through the activation of p38 kinase and JNK. This protein induce the recruitment of $\mathrm{HuR}$ (one of the $\mathrm{Hu}$ family proteins) and PAIP2 (poly(A)-binding proteininteracting protein 2) to the AU-rich elements (AREs) in the 3'-untranslated region (3' UTR) of VEGF mRNA [23]. Since the ARE sequence at the $3^{\prime}$ UTR of the VEGF mRNA is present in all the isoforms, there is a possibility that all isoforms have the same stability and that the differences observed in VEGF isoforms ratio were not due to a difference in mRNA stability. In fact, a study that correlates the VEGF144 up-regulation with glucose starvation, suggest that other mechanism apart mRNA stability must also exist to explain the dramatic increase observed (the increased stability observed for this isoform was approximately threefold but the increase in mRNA was 400-fold) [24]. Nevertheless, more studies have to be done to address this important question.

Additionally, the p38 pathway, which is an important stress signaling pathway that have been described to control VEGF at the mRNA expression [25] and stability level [22], was also shown to act directly or indirectly in the alternative splicing of this gene.

In conclusion, we postulate that changes of VEGF isoforms observed in acidic conditions may represent the adaptation of tumors to alterations in the microenvironment, namely by activating angiogenic signaling pathways, through different VEGF isoforms production.

\section{Material and Methods}

All reagents were obtained from Sigma, unless otherwise stated.
Cell Lines and Cell Culture Conditions

The RL95 cell line was kindly provided by Professor Steve Smith (currently Principal of the Faculty of Medicine, Imperial College, London, UK). It was cultured in 50\% high glucose Dulbecco's modified Eagle's medium (DMEM) medium (Sigma) and 50\% nutrient mixture F-12 Ham (Sigma), supplemented with $10 \%$ FBS, $100 \mu \mathrm{g} / \mathrm{ml}$ of streptomycin sulfate, $100 \mathrm{U} / \mathrm{ml}$ of penicillin $\mathrm{G}$ sodium, $2 \mathrm{mM}$ of L-glutamine and $0.1 \mu \mathrm{g} / \mathrm{ml}$ of amphotericin B as Fungizone. Cells were cultured at $37^{\circ} \mathrm{C}$ in a $5 \% \mathrm{CO}_{2}$ atmosphere.

Upon reaching confluency, RL95 cells were submitted to

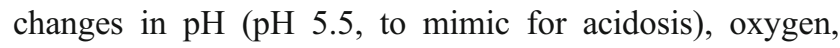
glucose $(100 \mathrm{mM})$ or hormones levels. To obtain an acidic medium we used $\mathrm{HCl}$ and the $\mathrm{pH} 5.5$ was confirmed before and after the experiment using a $\mathrm{pH}$ electrode. To induce hormonal changes and to mimic hypoxia, $\beta$-estradiol (100 $\mathrm{nM}) /$ progesterone $(1 \mu \mathrm{M})$ and cobalt chloride $(150 \mu \mathrm{M})$ [26] were used respectively.

To test the importance of the different signaling pathways in the regulation of VEGF alternative splicing, the RL95 cell line was cultured in growth medium at $\mathrm{pH} 5.5$ with or without the inhibitors of p38 MAPK (SB202190, Sigma, $20 \mu \mathrm{M}$,) and SAPK/JNK (SP600125, Sigma, $20 \mu \mathrm{M})$ signaling pathways.

RNA Isolation and Real Time RT-PCR with TAQMAN or Sybergreen

RNA was extracted by Trizol reagent (Invitrogen) and $2 \mu \mathrm{g}$ of total RNA was used to synthesize cDNA [27]. The mRNA level of each VEGF isoform was measured by real time RT-PCR (TAQMAN or Sybergreen) on the ABI Prism ${ }^{\circledR}$ 7900HT Sequence Detection System (Applied Biosystems) using specific primers and probes represented 
in Fig. 1 and Table 1. Expression of different SR proteins was analyzed with Sybergreen with specific primers (SF2/ ASF: forward-5'-GAA CAA CGA TTG CCG CAT CT-3', reverse-5'-AAT CAT AGC CGT CGC GAC C-3'; SRp20: forward-5'-GCA TCG TGA TTC CTG TCC ATT-3', reverse-5'-GTT CTT CCA TCT AGC TCT CGG ACT3'; SRp40: forward-5'-CTG TCG GGT ATT CAT CGG GA-3', reverse- $5^{\prime}$-ACG GTC AGA GTA TCG TCC TCT ACC T-3'). Real time PCR program consisted of an initial denaturation step at $95^{\circ} \mathrm{C}$ for $10 \mathrm{~min}$ followed by 40 cycles at $95^{\circ} \mathrm{C}$ for $15 \mathrm{~s}$ and at $60^{\circ} \mathrm{C}$ for $1 \mathrm{~min}$. The housekeeping gene used to normalize the samples in TAQMAN assay was the 18S (Human 18S rRNA-20×, Applied Biosystems). For Sybergreen, the BCR gene was used (forward- $5^{\prime}$ GAG CGT GCA GAG TGG AGG GAG AAC A-3'; reverse-5'-CAC AGT ATC CTC AGG GTC TGG GA-3').

The relative expression of each sample was calculated with respect to a standard calibration curve that represents a serial dilution of a cDNA. Each sample was analyzed in triplicate and each PCR experiment included at least one non-template control well.

\section{ELISA, Protein Extraction and Western Blotting}

Culture supernatants from RL95 in different conditions were collected and used to measure human VEGF by ELISA (Oncogene Research Products) under conditions described by the supplier.

To extract total proteins the pellets were suspended in a buffer containing 1\% NP40, 10\% glycerol, $50 \mathrm{mM}$ Tris$\mathrm{HCl} \mathrm{pH} 7.5,0.1 \%$ sodic azid and $150 \mathrm{mM} \mathrm{NaCl}$, supplemented with protease and phosphatase inhibitors. After $30 \mathrm{~min}$ in ice, lysates were centrifuged for $15 \mathrm{~min}$ at $4^{\circ} \mathrm{C}$ and $12,000 \mathrm{rpm}$.

Equal proteins amount were separated by sodium dodecyl sulphate-polyacrylamide gel electrophoresis (SDS-PAGE) and transferred to nitrocellulose membranes. Blots were incubated overnight with an antibody against phosphorilated SR proteins [mouse anti-SR proteins 1 (H4) from Zymed] at a concentration of $10 \mu \mathrm{g} / \mathrm{ml}$. Antibodies against P-AKT, PERK, P-JNK (Santa Cruz Biotechnology, Santa Cruz, CA, USA), P-p38 (cell signaling) and actin (Sigma) were used at final concentration of $100 \mathrm{ng} / \mathrm{ml}, 400 \mathrm{ng} / \mathrm{ml}$, and a dilution of 1:500, 1:1,000 and 1:2,000 respectively. Western blotting was performed following conventional protocols. Blots were revealed with the ECL (Amersham) system, according to manufacturers instructions. The hybridizing signals were quantified with ImageJ software.

siRNA for SR proteins

One day before transfection, $1 \times 10^{5}$ RL95 cells were plated in $500 \mu \mathrm{l}$ of growth medium without antibiotics in a $24-$ well culture vessel. Fifty picomoles of each siRNA (SFRS1 for SF2/ASF; SFRS3 for SRp20 and SFRS5 for SRp40 from Ambiom Company) were used. The RNA and $1 \mu 1$ of Lipofectamine 2000 (Invitrogen) were diluted separately in $50 \mu 1$ of Opti-MEM I reduced serum medium. After 5 min of incubation, the two dilutions were combined and incubated for $20 \mathrm{~min}$ at room temperature. This mixture was then added to the cells following an incubation of $6 \mathrm{~h}$ at $37^{\circ} \mathrm{C}$ and $5 \% \mathrm{CO}_{2}$. After transfection, the medium was replaced. The effect of transfection was assessed after $72 \mathrm{~h}$.

\section{Bioinformatics Approach/Program}

The splicing rainbow (Morais \& Valcarcel EMBL 2002 at http://www.ebi.ac.uk/asd-srv/wb.cgi?method=8) bioinformatics software was used to find putative binding sites for SF2/ASF, SRp20 and SRp40 proteins in the VEGF sequence. From the list of binding sites received after the VEGF sequence analysis we only choose as putative binding sites the sequences with higher scores (S) that were in exons. In detail, a sequence was considered a putative binding site for SRp20, and SF2/ASF if $S>6$ and for SRp40 if $S>5$.

\section{Statistical Analysis}

Results are expressed as mean \pm standard deviation. Data were analyzed using the unpaired two-tailed Student's $t$ test or the one-way ANOVA with post Tukey test. $p$ values of $<0.05$ were considered significant.

Acknowledgement We are grateful to Nuno Morais (PhD student, Unidade de Biologia Celular, Instituto de Medicina Molecular, Lisbon, Portugal) for his help in the bioinformatics analysis. We also thank Professor Steve Smith (currently Principal of the Faculty of Medicine, Imperial College, London, UK) for providing the RL95 cell line, and Mr. Alex Varey (Microvascular Research Laboratories, University of Bristol) for his useful suggestions regarding the $\mathrm{VEGF}_{\mathrm{xxx}} \mathrm{b}$ isoforms. Ana Paula Elias is a recipient of SFRH/BD/14287/2003 Fellowship (from the Portuguese Foundation for Science and Technology, FCT). This study was supported by POCTI 38391/2001 (Sérgio Dias) and by Liga Portuguesa Contra o Cancro, Nucleo Regional Sul.

\section{References}

1. Koukourakis MI, Pitiakoudis M, Giatromanolaki A et al (2006) Oxygen and glucose consumption in gastrointestinal adenocarcinomas: correlation with markers of hypoxia, acidity and anaerobic glycolysis. Cancer Sci 97:1056-1060

2. Sund M, Zeisberg M, Kalluri R (2005) Endogenous stimulators and inhibitors of angiogenesis in gastrointestinal cancers: basic science to clinical application. Gastroenterology 129:2076-2091

3. Sato Y (2006) Update on endogenous inhibitors of angiogenesis. Endothelium 13:147-155

4. Bergers G, Benjamin LE (2003) Tumorigenesis and the angiogenic switch. Nat Rev Cancer 3:401-410 
5. Tischer E, Mitchell R, Hartman T et al (1991) The human gene for vascular endothelial growth factor. Multiple protein forms are encoded through alternative exon splicing. J Biol Chem 266:11947-11954

6. Poltorak Z, Cohen T, Sivan R et al (1997) VEGF145, a secreted vascular endothelial growth factor isoform that binds to extracellular matrix. J Biol Chem 272:7151-7158

7. Nakamura M, Abe Y, Tokunaga T (2002) Pathological significance of vascular endothelial growth factor A isoform expression in human cancer. Pathol Int 52:331-339

8. Grunstein J, Masbad JJ, Hickey R et al (2000) Isoforms of vascular endothelial growth factor act in a coordinate fashion To recruit and expand tumor vasculature. Mol Cell Biol 20:72827291

9. Catena R, Muniz-Medina V, Moralejo B et al (2007) Increased expression of VEGF121/VEGF165-189 ratio results in a significant enhancement of human prostate tumor angiogenesis. Int $\mathrm{J}$ Cancer 120:2096-2109

10. Dent P, Yacoub A, Contessa J et al (2003) Stress and radiationinduced activation of multiple intracellular signaling pathways. Radiat Res 159:283-300

11. Graveley BR (2000) Sorting out the complexity of SR protein functions. RNA 6:1197-1211

12. Bates DO, Cui TG, Doughty JM et al (2002) VEGF165b, an inhibitory splice variant of vascular endothelial growth factor, is down-regulated in renal cell carcinoma. Cancer Res 62:4123-4131

13. Wu JY, Maniatis T (1993) Specific interactions between proteins implicated in splice site selection and regulated alternative splicing. Cell 75:1061-1070

14. Fayette J, Soria JC, Armand JP (2005) Use of angiogenesis inhibitors in tumour treatment. Eur J Cancer 41:1109-1116

15. Ferrara N (1995) The role of vascular endothelial growth factor in pathological angiogenesis. Breast Cancer Res Treat 36:127-137

16. Vlachtsis K, Brieger J, Kim DW et al (2002) Quantitative analysis of VEGF-isoforms in head and neck squamous cell carcinoma cell lines: relation to xenotransplantability and tumour progression in mice. Oncol Rep 9:1133-1138
17. Zhang HT, Scott PA, Morbidelli L et al (2000) The 121 amino acid isoform of vascular endothelial growth factor is more strongly tumorigenic than other splice variants in vivo. $\mathrm{Br} \mathrm{J}$ Cancer 83:63-68

18. Fenton BM, Paoni SF, Liu W et al (2004) Overexpression of VEGF121, but not VEGF165 or FGF-1, improves oxygenation in MCF-7 breast tumours. Br J Cancer 90:430-435

19. Nishi M, Abe Y, Tomii $Y$ et al (2005) Cell binding isoforms of vascular endothelial growth factor-A (VEGF189) contribute to blood flow-distant metastasis of pulmonary adenocarcinoma. Int $\mathrm{J}$ Oncol 26:1517-1524

20. Fukumura D, Xu L, Chen Y et al (2001) Hypoxia and acidosis independently up-regulate vascular endothelial growth factor transcription in brain tumors in vivo. Cancer Res 61:6020-6024

21. Goerges AL, Nugent MA (2003) Regulation of vascular endothelial growth factor binding and activity by extracellular $\mathrm{pH}$. J Biol Chem 278:19518-19525

22. Pagès G, Berra E, Milanini J et al (2000) Stress-activated protein kinases (JNK and p38/HOG) are essential for vascular endothelial growth factor mRNA stability. J Biol Chem 275:26484-26491

23. Onesto C, Berra E, Grepin R, Pages G (2004) Poly(A)-binding protein-interacting protein 2, a strong regulator of vascular endothelial growth factor mRNA. J Biol Chem 279:34217-34226

24. Zhang L, Conejo-Garcia JR, Yang N et al (2002) Different effects of glucose starvation on expression and stability of VEGF mRNA isoforms in murine ovarian cancer cells. Biochem Biophys Res Commun 292:860-868

25. Tanaka T, Kanai H, Sekiguchi K et al (2000) Induction of VEGF gene transcription by IL-1 beta is mediated through stressactivated MAP kinases and Sp1 sites in cardiac myocytes. J Mol Cell Cardiol 32:1955-1967

26. Chandel NS, Maltepe E, Goldwasser E et al (1998) Mitochondrial reactive oxygen species trigger hypoxia-induced transcription. Proc Natl Acad Sci U S A 95:11715-11720

27. Roos-van Groningen MC, Eikmans M, Baelde HJ et al (2004) Improvement of extraction and processing of RNA from renal biopsies. Kidney Int 65:97-105 